\title{
Sex Steroids, Carcinogenesis, and Cancer Progression
}

\author{
LUIGI CASTAGNETTA, ${ }^{a, b}$ ORAZIA M. GRANATA, ${ }^{b}$ LETIZIA COCCIADIFERRO,${ }^{b}$ \\ ANNALISA SAETTA, ${ }^{b}$ LUCIA POLITO,${ }^{b}$ GIUSEPPE BRONTE, ${ }^{a}$ \\ SERGIO RIZZO,${ }^{a}$ ILDEGARDA CAMPISI, ${ }^{b}$ BIAGIO AGOSTARA,${ }^{c}$ \\ AND GIUSEPPE CARRUBA $^{a}$ \\ ${ }^{a}$ Department of Experimental Oncology and Clinical Application, University Medical \\ School, Palermo, Italy \\ ${ }^{b}$ Experimental Oncology Unit and ${ }^{d}$ Division of Medical Oncology, Department of Clinical \\ Oncology, ARNAS-Civico, M. Ascoli Cancer Hospital Center, Palermo, Italy
}

\begin{abstract}
The relationship between sex steroids and cancer has been studied for more than a century. Using an original intact cell analysis, we investigated sex steroid metabolism in a panel of human cancer cell lines, either hormone responsive or unresponsive, originating from human breast, endometrium, and prostate. We found that highly divergent patterns of steroid metabolism exist and that the catalytic preference (predominantly reductive or oxidative) is strictly associated with the steroid receptor status of cells. We explored intratissue concentrations and profiles of estrogens in a set of human breast tumors as compared to normal mammary tissues, also in relation to their estrogen receptor status. In particular, we showed that, with hydroxyestrogens representing the majority of all tissue estrogens, concentrations of individual metabolites, as well as their ratios, significantly differ when comparing normal tissue with cancer tissues or when they are related to the overall survival of cancer patients.
\end{abstract}

KEYWORDS: sex steroids; breast cancer; intratumor estrogens

\section{INTRODUCTION}

The relationship between sex steroids and cancer has been studied for more than a century. In the last decade, there have been a number of milestone achievements, especially in the study of their mechanism of action, receptors, and interaction with other signaling systems. Recently, two major issues have emerged: (1) The number of target tissues and cells identified for gonadal steroids has enormously increased over the last few years, to such a point that it has become easier to enumerate those tissues where no evidence has been provided thus far for a direct action of steroids.

Address for correspondence: Giuseppe Carruba, M.D., Ph.D., Department of Experimental Oncology and Clinical Application, University Medical School, M. Ascoli Cancer Hospital Center, ARNAS-Civico, Via C. Lazzaro 2, 90127 Palermo, Italy. Voice: +39-091-666-4346; fax: +39-091-666-4352.

lucashbl@unipa.it

Ann. N.Y. Acad. Sci. 1024: 233-246 (2004). (C) 2004 New York Academy of Sciences. doi: 10.1196/annals.1321.028 
(2) It is now recognized that steroid action goes far beyond the classical receptormediated mechanism. Steroid actions are also based upon rapid, membrane-bound responses and an intricate network of interaction and crosstalk with different intracellular signaling pathways. On the other hand, the potential role of gonadal steroids, especially estrogens, in both development and progression of human endocrine-related tumors remains unresolved. The seeming inconsistency that these tumors have a higher incidence in aged subjects, just when a steady decline of steroid formation rates and circulating levels occurs, remains troubling. This apparent contradiction has been partly unraveled by studies indicating that steroid target tissues (specifically breast, endometrium, and prostate) are endowed with an assorted repertoire of steroid enzymes and are able to locally produce and accumulate biologically active steroid metabolites. This may eventually lead to intratissue amounts of steroids 10 to 100 times greater then those found in plasma-even in subjects aged 50 years and above. In addition, a deeper understanding of the carcinogenesis process indicates that, depending on the tumor type, 20 to 50 years may be required to complete the phenotypical and genotypical transformation of a single initiated cell, hence accounting for the higher cancer incidence rates found in older ages.

Our own research group studied the role of sex steroids in hormone-related tumors, notably breast, endometrial, and prostate cancer. In 1977, we first noted that breast cancer patients have urinary levels of estrogens significantly higher than those found in healthy postmenopausal women. ${ }^{1}$ Further study revealed that "unusual" estrogen metabolites (more polar derivatives as opposed to classical estrogens) represent the majority of urinary estrogens in breast cancer patients. ${ }^{2}$ Subsequently, we showed that unusual metabolites are excreted in markedly greater amounts in endometrial cancer patients whose tumors are estrogen receptor (ER)-negative. ${ }^{3}$ Using an original intact cell analysis, ${ }^{4}$ we investigated sex steroid metabolism in a panel of human cancer cell lines, either hormone responsive or unresponsive, originating from human breast, endometrium, and prostate. We found that highly divergent patterns of steroid metabolism exist and that the catalytic preference (predominantly reductive or oxidative) is strictly associated with the steroid receptor status of cells. ${ }^{5-7}$ More recently, we have explored intratissue concentrations and profiles of estrogens in a set of human breast tumors as compared to normal mammary tissues, also in relation to their ER status. ${ }^{8}$ In particular, we showed that, with hydroxyestrogens representing the majority of all tissue estrogens, concentrations of individual metabolites, as well as their ratios, significantly differ when comparing normal tissue with cancer tissues or when they are related to the overall survival of cancer patients.

In the following sections, we review data from our own in vivo and in vitro studies on sex steroid concentrations and metabolism in target tissues and cells, and provide a better understanding of the potential role of gonadal steroids, especially estrogens, in both cancer development and progression.

\section{INTRATISSUE ESTROGENS}

In a recent report, ${ }^{8}$ we inspected intratissue amounts and profiles of estrogens in a set of human breast tumors (20 cases) as compared to normal mammary tissues obtained from healthy women who underwent surgery for reduction mammoplasty 

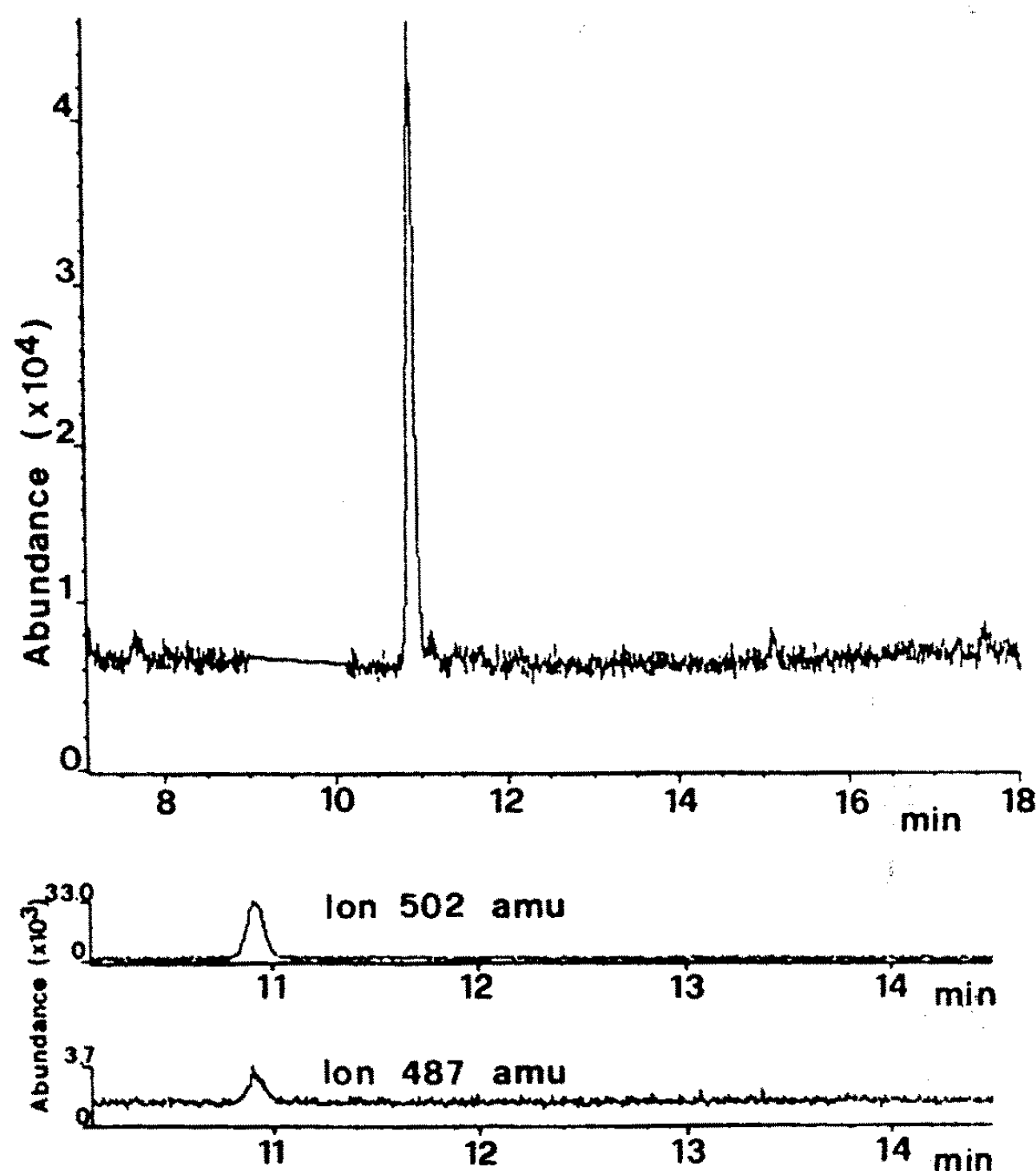

FIGURE 1. Selected ion gas chromatograph of the purified peak fraction from a breast tumor tissue extract. Ions monitored at $\mathrm{m} / \mathrm{z} 502(\mathrm{M}+)$ and $487(\mathrm{M}-15)+$ for the 2OHE1 estrogen derivative. (Reproduced with permission from Castagnetta et al. ${ }^{16}$ )

(6 cases). Although variable concentrations of hydroxylated estrogens were detected, they consistently accounted for more than $80 \%$ of all estrogens. Furthermore, various hydroxyestrogens showed significantly different amounts when comparing normal and malignant human breast tissues. Identity and amounts of individual estrogen derivatives were also authenticated using gas chromatography/mass spectrometry (GC/MS), as reported elsewhere. A typical GC/MS profile of $2 \mathrm{OHE} 1$ is shown in FIGURE 1.

In this study, concentrations of $16 \alpha \mathrm{OHE} 1$ were nearly 3.8 -fold higher in cancerous tissues than in normal tissues, even though this difference was not significant $(P=0.07)$, presumably because of the limited number of cases included in the study (FIG. 2). Conversely, 2OHE1 levels were almost equivalent in normal and cancer tissues, while its methoxy derivative, $2 \mathrm{MeOE} 1$, was found in significantly greater amounts $(P=0.024)$ in malignant tissues (FIG. 3). Interestingly, 4OHE1 and 4OHE2 


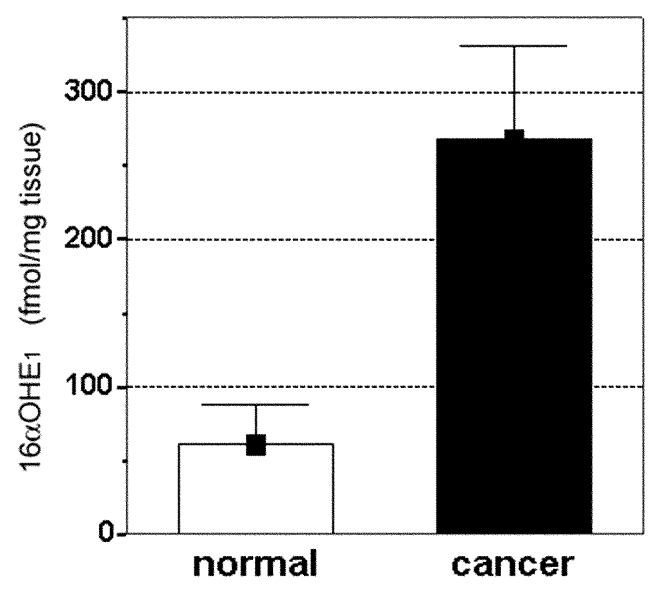

FIGURE 2. Intratissue concentrations of $16 \alpha \mathrm{OHE} 1$ in normal and malignant human breast tissues.
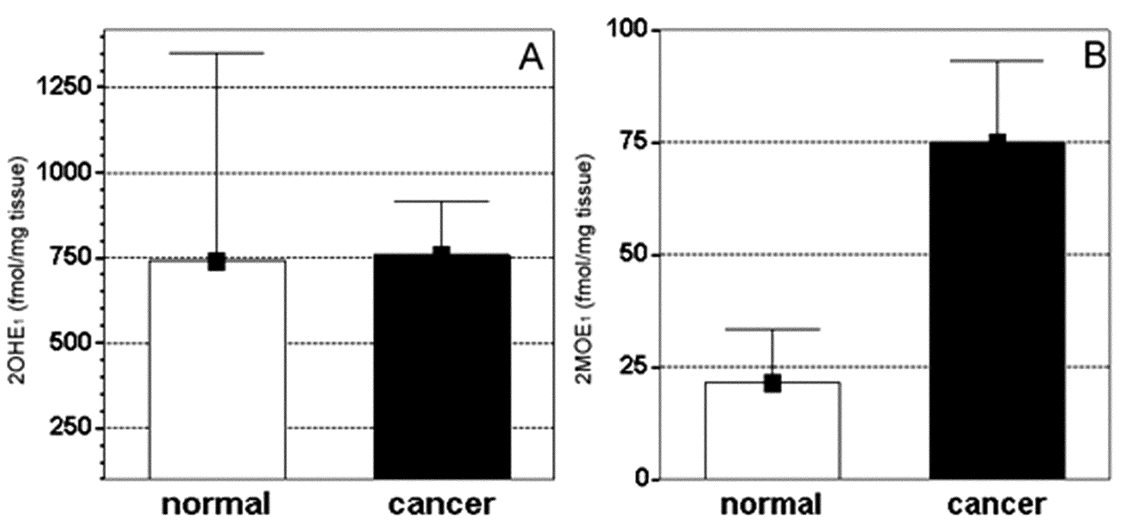

FIGURE 3. Intratissue concentrations of 2OHE1 (A) and 2MeOE1 (B) in normal and malignant human breast tissues.

levels were strikingly different in normal versus cancer tissues, with the former being markedly higher and the latter significantly greater in normal and malignant breast tissues, respectively (FIG. 4). The 4OHE2:4OHE1 ratio was found to be $<0.1$ in normal and $>3$ in cancer tissues, suggesting that 4-hydroxylase activity on E2 and E1 is differentially regulated. As far as 4-methoxy derivatives of both E1 and E2 are concerned, an equivalent situation occurs, with 4MeOE1 being elevated in normal and $4 \mathrm{MeOE} 2$ in cancer tissues (FIG. 5). It should be emphasized that methoxy derivatives of both estrone (E1) and estradiol (E2) are in far lower amounts than their parent hydroxyestrogens, except for normal cases, where the ratio of 4MeOE2 to 4OHE2 is greater than 1.6. Furthermore, 4OHE2 levels in cancer tissues were around eight times higher than in normal tissues and sevenfold greater than those of intratumor $16 \alpha \mathrm{OHE} 1$. As reported in TABLE 1 , the ratio of $2 \mathrm{OHE} 1: 16 \alpha \mathrm{OHE} 1$ changes dramatically in malignant tissues, dropping to 1.5 from 8.3 in normal breast tissues. This is exclusively the result of the significant rise of $16 \alpha \mathrm{OHE} 1$ in cancer cases (nearly fourfold), since 2OHE1 levels remain almost equivalent in normal and malignant tissues. Interestingly, ratios of 4-OHE1 to 2-OHE1 and OHE2, as well as 

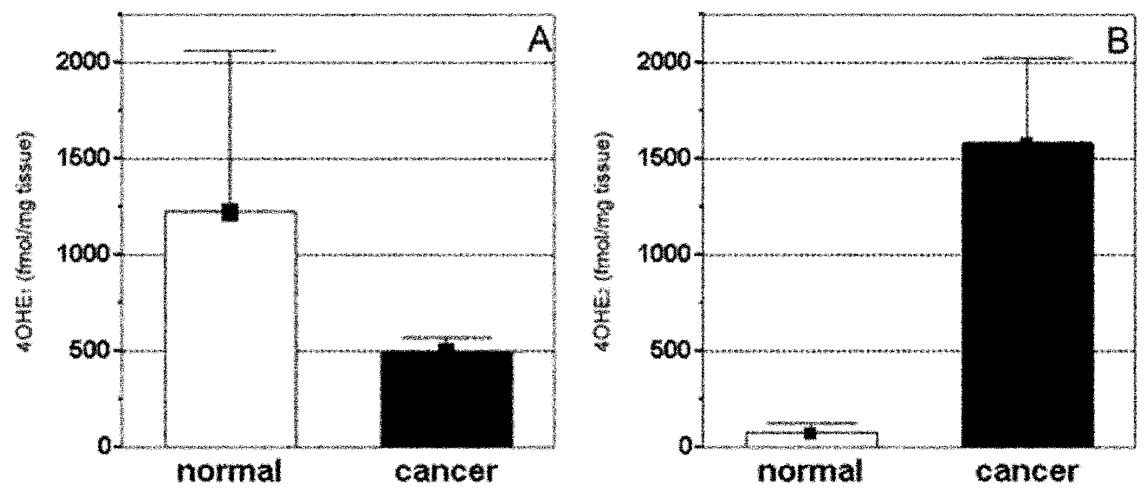

FIGURE 4. Intratissue concentrations of 4OHE1 (A) and 4OHE2 (B) in normal and malignant human breast tissues.
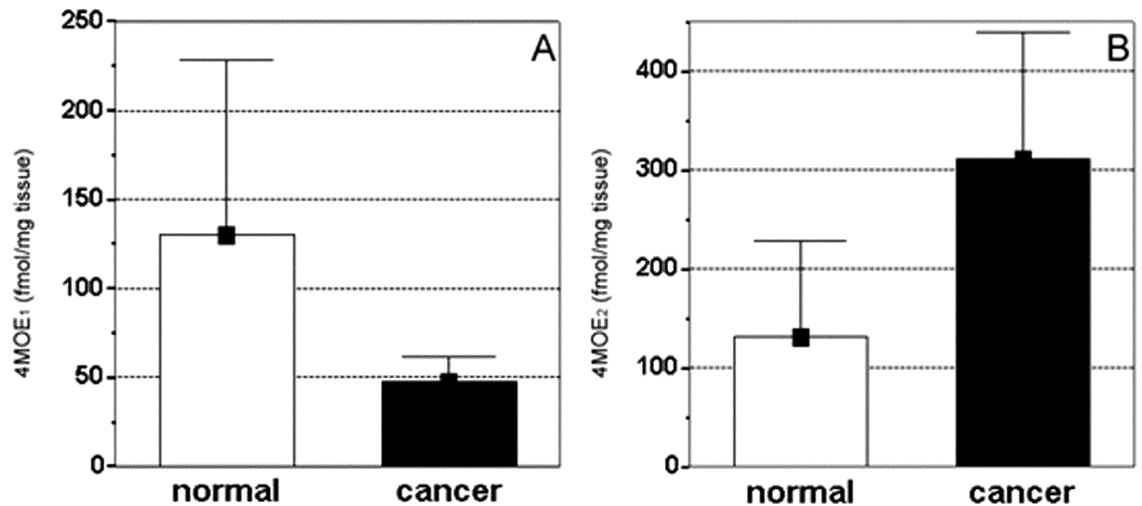

FIGURE 5. Intratissue concentrations of 4MeOE1 (A) and 4MeOE2 (B) in normal and malignant human breast tissues.

TABLE 1. Ratio values of hydroxyestrogens in normal and malignant human breast tissues

\begin{tabular}{lcccc}
\hline \multicolumn{1}{c}{$\begin{array}{c}\text { Breast } \\
\text { tissue }\end{array}$} & OHE1 & OHE1 & OHE2 & OHE1/OHE2 \\
\hline Normal $(N=6)$ & $8.32 \pm 4.58$ & $44.61 \pm 42.25$ & $1.45 \pm 0.47$ & $60.91 \pm 41.92$ \\
Cancer $(N=17)$ & $1.54 \pm 0.11$ & $1.56 \pm 0.77$ & $13.82 \pm 9.78$ & $4.43 \pm 1.98$ \\
$t$ test & $P=0.016$ & $P=0.087$ & $P=0.464$ & $P=0.028$ \\
\hline
\end{tabular}

NotE: Values are expressed as mean $\pm \mathrm{SE}$. 
those of 4OHE1 to 4OHE2, reveal a significant difference when comparing normal with cancerous breast samples (TABLE 1). In particular, values of 4OHE1:2OHE1 and 4OHE2:2OHE2 ratios were markedly greater in normal and in malignant breast tissues, respectively, implying that $4 \mathrm{OHE} 1$ is prevalent in normal tissues, while 4OHE2 is predominant in cancer cases. This eventually led the 4OHE1:4OHE2 ratio to be significantly $(P=0.028)$ higher in normal than in cancer tissues (TABLE 1).

Relevant literature on intratissue amounts of estrogens in human breast is somewhat scarce and inconsistent. Our data are in agreement with those of Cavalieri and colleagues, ${ }^{9}$ although in our work concentrations of both classical (E2, E1, E3) and hydroxy estrogens are found to be markedly greater (10 to 50 times) than those reported in Cavalieri's paper. This seeming inconsistency may be explained on the basis of the different methodological approaches used for estrogen extraction and chromatography, our recovery values being generally high (65-95\%) and frequency of detection for classical and hydroxylated estrogens being generally higher with respect to that obtained by Cavalieri and colleagues (our study: normal breast, 3467\%; malignant breast, 65-98\%. Cavalieri's study: normal breast, 10-43\%; malignant breast, $18-57 \%$ ).

\section{IN VITRO STUDIES}

There is concurrent evidence in the literature that distinct metabolic patterns of either estrogen or androgen are encountered in cultured human cancer cell lines derived from the main endocrine-related tumors (such as breast, endometrium, and prostate), depending on their steroid receptor status and response to hormones. ${ }^{5-7,10,11}$ This different metabolic aptitude mostly relates to the activity of the $17 \beta$-hydroxysteroid dehydrogenase (17 $\beta$ HSD) enzyme system and is reflected in the prevalence of reductive metabolism in the receptor-positive, hormone-responsive cells; while oxidative pathways are largely dominant in receptor-negative, unresponsive cells. This figure is further refined by the evidence that, in addition to $17 \beta \mathrm{HSD}$, different steroid enzyme activities, such as sulfotransferase/sulfatase and hydroxylases, are significantly associated with both receptor levels and response to estrogen in target cancer tissues and cells. $^{12-15}$

In our own studies we have adopted an original intact cell analysis (see following section) to investigate rates and patterns of estrogen metabolism in a panel of established human cancer cell lines, either hormone responsive (e.g., MCF7, ZR75-1, T47D) or unresponsive (e.g., MDA-MB-231, BT20, HBL100), from the human breast. In particular, using this approach, we were able to assess formation and degradation of various hydroxy estrogens, especially catechol estrogens (CCEs) and their methoxy $(\mathrm{MeO})$ derivatives. The major metabolic pathways eventually leading to formation of estrogens and their hydroxy or methoxy derivatives are portrayed in FIGURE 6. FIGURE 7 illustrates a typical chromatographic profile of estrogen metabolism in MCF7 human breast cancer cells.

In vitro data obtained in our laboratories clearly indicate that ER-positive breast tumor cells privilege reductive pathways of estrogen metabolism leading to E2 accumulation, exactly as it occurs in vivo in ER-positive tumor tissues. On the other hand, ER-negative cells preferentially oxidize E2 to E1, which is not further metabolized, and (as in ER-negative cancer tissues) accumulates as such. These results are 


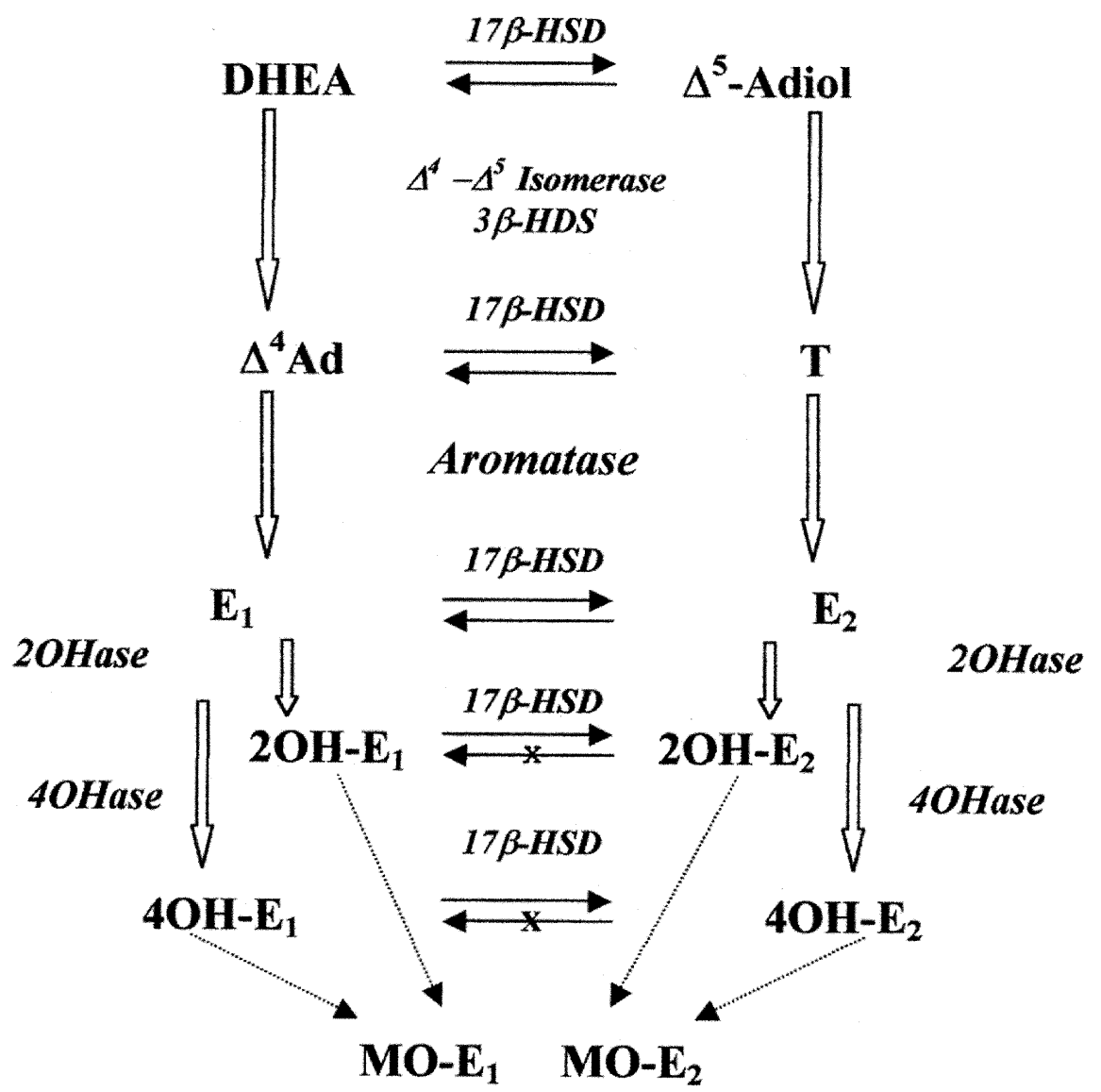

FIGURE 6. Flow chart of major metabolic pathways that control formation of estrogens and their hydroxy and methoxy derivatives.

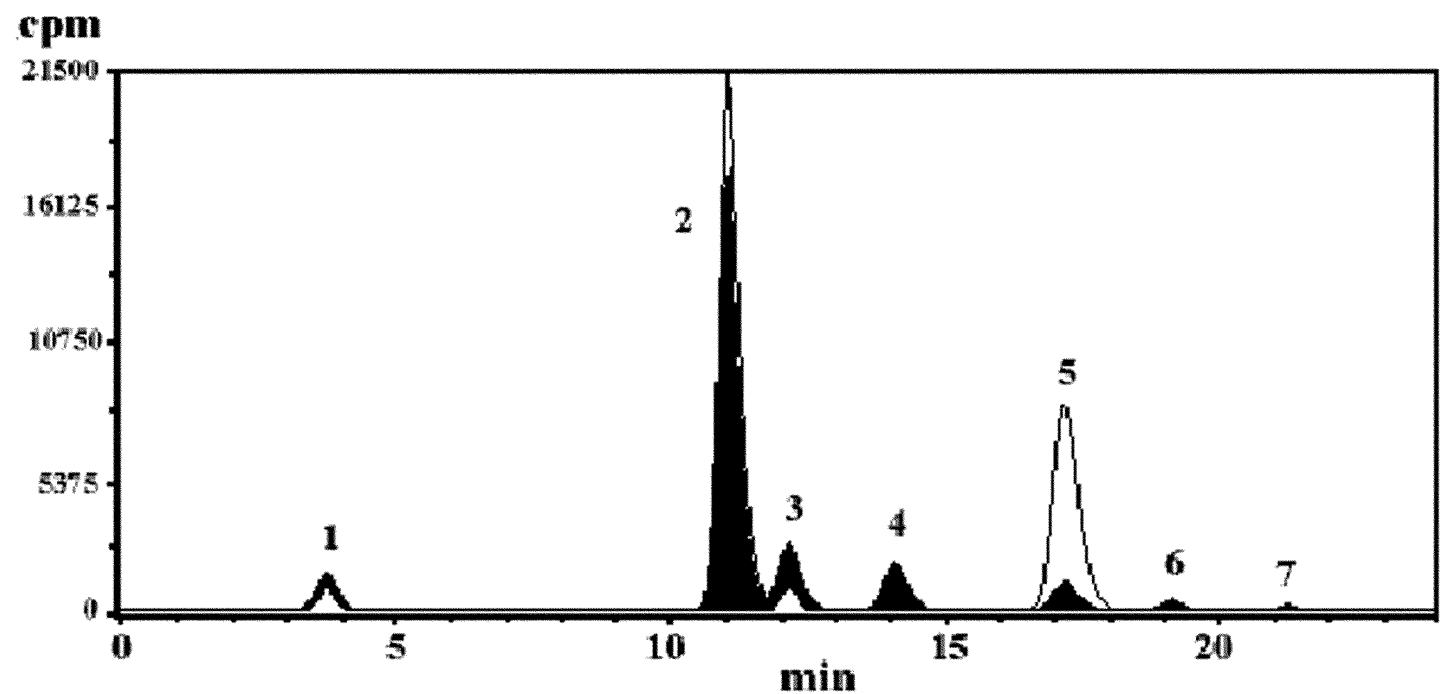

FIGURE 7. A typical HPLC profile of estrogen metabolism in MCF7 breast cancer cells incubated for $72 \mathrm{~h}$ with tritiated estradiol. White and black peaks indicate free $(\mathrm{F})$ and sulfate (S) estrogens, respectively. Percent conversion rates for individual peaks were: (1) E3: F, 1.03\%; S, 1.10\%. (2) E2: F, 36.46\%; S, 31.44\%. (3) 4MeOE2: F, 1.19\%; S, 5.00\%. (4) 2MeOE2: F, ND; S, 3.70\%. (5) E1: F, 16.69\%; S, 2.26\%. (6) 4MeOE1: F, 0.21\%; S, $0.42 \%$. (7) 2MeOE1: F, ND; S, 0.09\%. ND, not detectable. 
TABLE 2. Formation rates of hydroxy and methoxy estrogens in MCF-7 and ZR75-1 human breast cancer cells

\begin{tabular}{cccc}
\hline Cell & $2 \mathrm{OH}+2 \mathrm{MeO}$ & $4 \mathrm{OH}+4 \mathrm{MeO}$ & $4 / 2$ Ratio \\
\hline MCF-7 & $2.30 \pm 0.09$ & $2.12 \pm 0.05$ & $0.92 \pm 0.02$ \\
ZR75-1 & $2.09 \pm 0.10$ & $0.20 \pm 0.01$ & $0.10 \pm 0.01$ \\
\hline
\end{tabular}

NotE: Cells were incubated for 24 hours with tritiated E2 as precursor. Values represent mean $\pm \mathrm{SD}$ of sum of free and conjugate metabolites of E2 from three separate experiments.

TABLE 3. Catechol- and methoxyestrogen formation in MCF7 cells after 72-h incubation with estradiol (E2) or estrone (E1) as precursor

\begin{tabular}{lcc}
\hline & \multicolumn{2}{c}{ Tritiated precursor } \\
\cline { 2 - 3 } & ${ }^{3} \mathrm{H}-\mathrm{E} 2$ & ${ }^{3} \mathrm{H}-\mathrm{E} 1$ \\
\hline $2 \mathrm{OH}+2 \mathrm{MeO}$ & $1.49 \pm 0.09$ & $0.70 \pm 0.05$ \\
$4 \mathrm{OH}+4 \mathrm{MeO}$ & $2.66 \pm 0.21$ & $1.14 \pm 0.07$ \\
$4 / 2$ ratio & $1.78 \pm 0.08$ & $1.63 \pm 0.08$ \\
\hline
\end{tabular}

NotE: Values represent mean pmoles \pm SD of sum of free and conjugate metabolites from three separate experiments.

largely at variance with those obtained using a crude-extract approach, in both quantitative and qualitative terms. In fact, while the overall 17ßHSD activity, as measured by intact cell analysis, is markedly lower than that expected on the basis of classical enzymology, the extent of the oxidative component of 17ßHSD diverges drastically using the two different approaches, being significantly greater in ER-negative mammary cancer cells or in ER-positive breast tumor tissues, respectively (data not shown).

In hormone-responsive cells, including MCF7 and ZR75-1, we observed an early production of either free or conjugated CCE after a 24-h incubation with a tritiated estrogen precursor, either E2 or E1. In particular, appreciable amounts of free 2OHE2, 2MeOE1, and 2MeOE2, as well as of conjugate 2MeOE2, were consistently detected in both cell lines, leading to nearly $5 \%$ formation of both hydroxy and methoxy estrogens in MCF7 cells and 2.3\% in ZR75-1 cells (TABLE 2). After 72-h incubation, however, hydroxy estrogen production increased considerably, revealing the formation of almost all major CCE and methoxy compounds (including 2OHE2, 2MeOE1, 2MeOE2, and 4MeOE1). 4MeOE2 was the prevalent metabolite among either free or conjugate estrogens (not shown). From these experiments, there is also consistent evidence that products of 4-hydroxylation (including both hydroxy and methoxy compounds) are predominant over those of 2-hydroxylation, eventually causing the ratio of 4-hydroxy estrogens to 2-hydroxy estrogens to be relatively high in these cell model systems (TABLE 3). In addition, formation of CCE and methoxy derivatives from E2 is in MCF7 cells twofold greater than that observed using E1 as precursor (TABLE 3). This evidence may also be explained on the basis of a prevalently reductive activity of the 17ßHSD enzyme in MCF7 cells, leading to accumu- 
TABLE 4. Hydroxyestrogen formation in MCF-7 after 24-h and 72-h incubation with androstenedione as precursor

\begin{tabular}{ccc}
\hline Hours & 4OHE2 & 2OHE1 \\
\hline 24 & $0.93 \pm 0.08$ & $0.41 \pm 0.03$ \\
72 & $3.92 \pm 0.10$ & $0.69 \pm 0.06$ \\
\hline
\end{tabular}

NotE: Values represent mean pmoles \pm SD from three different experiments.

lation of E2 and its hydroxy derivatives. Conversely, in ZR75-1 cells, where the reductive $17 \beta \mathrm{HSD}$ activity is lower, the overall CCE formation rates are reduced and ratios of 2-hydroxy to 4-hydroxy derivatives are changed (not shown).

The early formation of CCE in both MCF7 and ZR75-1 cells indicates that estrogen hydroxylation may represent an important metabolic pathway, while the prevalence of 4-hydroxy derivatives over 2-hydroxy derivatives is in accordance with our in vivo data on intratissue estrogens. ${ }^{8}$ In separate experiments we have also inspected estrogen formation in human breast cancer cells using tritiated androstenedione (Ad) as an androgen precursor to assess the extent of aromatase activity on Ad, which represent a major source of estrogen production in postmenopausal women. Results from these studies clearly reveal a consistent CCE production after 24- and 72-h incubations (up to nearly $10 \%$ by 72 hours), with 4OHE2 and 2OHE1 being the two most prevalent hydroxyestrogens (TABLE 4).

\section{INTACT CELL ANALYSIS}

Different methodological procedures have been used to measure key enzyme activities of sex steroid metabolism, either in vivo or in vitro. Unfortunately, results from these studies diverge substantially. First, there is a lack of correlation between tissue levels of endogenous hormones and both extent and direction of steroid metabolism, as measured by the classical enzymology approach. It should be pointed out that while some earlier studies used conditions that approximate the in vivo state (fresh tissue slices unsupplemented with pyridine-nucleotide cofactors), the majority measured enzyme activities in more artificial environments (tissue homogenates supplemented with NADPH as cofactor), which overlook the regulatory mechanisms existing in intact cells and neglect the endogenous cofactors.

It should be emphasized that, from a methodological standpoint, measurement of an enzyme system, a complex and dynamic structure, using crude cell and/or tissue extracts and classical enzymology methods is doubtless far more artificial and puzzling than the quantitation of individual steroids in tumor tissue homogenates. Quantitative estimates of the enzyme kinetic data $\left(K_{\mathrm{m}}\right.$ and $\left.V_{\max }\right)$ are currently carried out under artificial conditions (cell lysates, $\mathrm{pH}$, temperature, excess of substrate concentration, cofactor addition, and so forth) and optimized to yield the maximum conversion rates for quantitation of a single enzyme activity. This condition largely differs from physiological environment of $\mathrm{pH}$, temperature, cofactor, substrate concentration, and enzyme inhibitors in vivo. Hence, classical enzymology data are highly unlikely to be representative of the in vivo condition. 
TABLE 5. Major advantages of intact cell analysis and disadvantages of the classical enzymology approach to measuring the activity of key steroid enzymes

Advantages of intact cell analysis

Simultaneous inspection of multiple enzyme activities in living, cultured cells using physiological amounts of a steroid precursor

Assessment of rates and direction of steroid metabolism by continuous monitoring of both precursor degradation and product formation, avoiding artificial variation of cellular microenvironment

Disadvantages of classical enzymology

Disregards the regulatory mechanism existing in intact cells and neglects the endogenous cofactors

Quantitatively estimates enzyme kinetic data $\left(K_{\mathrm{m}}\right.$ and $\left.V_{\max }\right)$ under conditions optimized to yield maximum conversion rates with artificial $\mathrm{pH}$, temperature, cofactor, and substrate concentration

No appraisal of the extent and direction of steroid metabolism

No correlation with intratissue amounts of endogenous steroids

TABLE 6. Efficiency values of radiometric detection of tritiated estradiol at continuous flow in standard conditions

\begin{tabular}{ccc}
\hline dpm (theoretical) & cpm (observed) & Efficiency $(\%)$ \\
\hline 294 & 52 & 18 \\
587 & 81 & 14 \\
1174 & 192 & 16 \\
2349 & 433 & 18 \\
4699 & 987 & 21 \\
9318 & 1524 & 16 \\
18,798 & 3097 & 16 \\
37,593 & 6125 & 16 \\
75,187 & 13,463 & 18 \\
\hline
\end{tabular}

Note: dpm, decline/minute; cpm, count/minute.

TABLE 7. Detection limits and efficiency values of radiometric detection online to RP-HPLC

\begin{tabular}{cccc}
\hline & \multicolumn{2}{c}{ Detection limits } & \\
\cline { 2 - 3 } Compound & Moles & $\mathrm{cpm}^{a}$ & Efficiency (\%) \\
\hline$\left[4-{ }^{14} \mathrm{C}\right] \mathrm{E} 1$ & $1.1^{b}$ & $70.2 \pm 4.1^{c}$ & $56.2 \pm 5.2^{c}$ \\
{$\left[6,7-{ }^{3} \mathrm{H}\right] \mathrm{E} 2$} & $2.4^{d}$ & $51.3 \pm 2.1^{c}$ & $17.3 \pm 1.2^{c}$ \\
\hline
\end{tabular}

${ }^{a}$ Counts per minute.

${ }^{b}$ Picomoles.

${ }^{c}$ Values represent mean $\pm \mathrm{SD}$ of six different analyses; processing cell culture medium spiked with pure radiolabeled compounds.

${ }^{d}$ Femtomoles. 
Aiming to assess rates and pathways of sex steroid metabolism, either in vivo or in vitro, we originally developed, established, and optimized a novel approach we named intact cell analysis. ${ }^{4,16}$ In this method, the use of reverse-phase high performance liquid chromatography (RP-HPLC) with online radioactive detection allows a simultaneous and sequential estimation of several enzyme activities in intact cultured cells or tissue fragments incubated for variable time lengths (from a few minutes up to several days) with physiological amounts of a radioactive (tritiated) sex steroid used as precursor. Using this approach, we could analyze, under strictly controlled experimental conditions, the rates and direction of steroid conversion to a wide range of either conjugate or unconjugated metabolites (up to 25) in a relatively short time of analysis (20-30 minutes). This "live analysis" system permits continuous monitoring and quantitation of both precursor degradation and formation of several metabolic products; it avoids the artifacts of classically measured enzyme reactions and, notably, obtains more refined information on the metabolic direction in individual tissues and cells. The advantages of this methodology, as opposed to the disadvantages of classical enzymology approach, are summarized in TABLE 5.

It is noteworthy that radiometric detection online to HPLC is featured by high efficiency values (TABLE 6) and low detection limits (TABLE 7). This, combined with the good linearity of this approach (FIG. 8), permits detection limits as low as 2.4 fmoles and 1.1 pmoles for pure tritiated E2 and pure carbonated E1, respectively (TABLE 8). In addition, the use of the aluminum oxide acid (alumina) absorption extraction method markedly improved recovery values of CCEs, hence further increasing the sensitivity of this procedure. This represents an essential prerequisite to our approach for studying metabolic pathways of gonadal steroids, either in vivo or in vitro, where target tissues or cells are exposed to physiological concentrations of a labeled precursor.

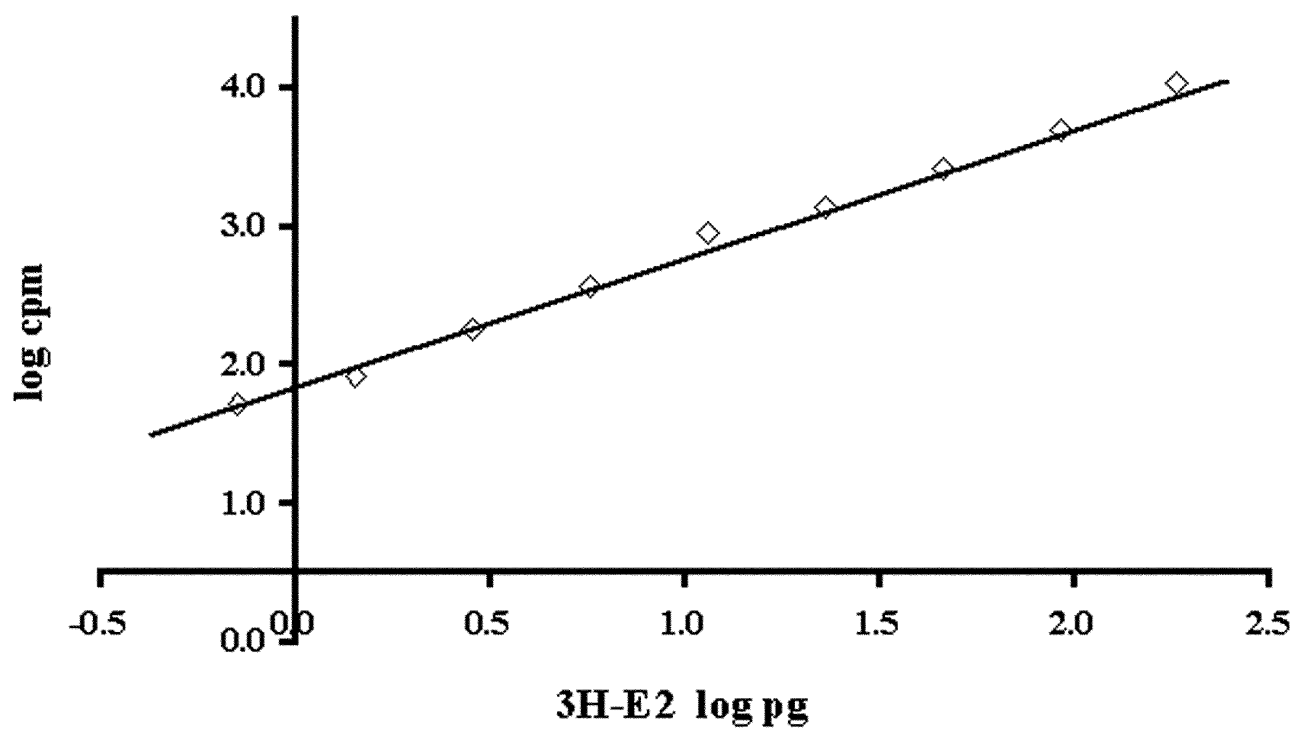

FIGURE 8. Linearity of radiometric detection online to RP-HPLC for ${ }^{3} \mathrm{H}$-E2. Regression equation: $y=1.824+0.968 x$. Correlation coefficient $=0.994 . P<10^{-6}$, Pearson's test. 
TABLE 8. Recovery values for selected catecholestrogens using the alumina adsorption extraction method

\begin{tabular}{cc}
\hline Compound & Recovery $(\%)$ \\
\hline 2OHE1 & $65-75$ \\
E1 & $65-72$ \\
2MeOE1 & $84-93$ \\
\hline
\end{tabular}

It might be argued that intact cell analysis cannot be considered entirely representative of tissue dynamics in vivo. Intact cell analysis does, however, more closely approach the in vivo state than the artificial requirements of classical enzymology procedures. Of course, one should place very wide confidence intervals in extrapolating data obtained in vitro to the in vivo situation. In particular, the absence of the stromal compartment should be carefully considered. However, some evidence seems to corroborate results gained on cultured epithelial cancer cells. Preliminary data obtained in our laboratories on primary cultures of stromal cells isolated from primary breast tumor tissues appears to indicate that the stromal contribution to the overall rates and direction of estrogen metabolism is relatively trivial (Castagnetta and colleagues, in preparation). This would imply that the metabolic fate of circulating estrogens is mostly subjected to the metabolic behavior of tumor epithelial cells and that expression and activity of key steroid enzymes in individual tissues and cells may lead to local accumulation of more-or-less biologically active metabolites.

\section{CONCLUSIONS}

Fifteen years ago, on the occasion of an international meeting we held in Taormina (Sicily), we first introduced a new concept in the endocrinology of human malignancies - the peripheral formation, degradation, and action of sex steroids, which suggested that hormonal regulation of target cancer cells may be directed primarily by the local expression and activity of key enzymes in steroid metabolism. ${ }^{17}$ This concept was further elaborated by Fernand Labrie, who coined the term intracrinology to define hormone effects whereby both the ligand and its receptor interact within the target cell. ${ }^{18}$

The evidence we present here suggests that estrogens may be implicated as initiators, not just as promoters, in the carcinogenic process (see the paper by Cavalieri and Rogan, this volume). In particular, our finding that the so-called minor estrogens, including CCE, other hydroxyestrogens ( $16 \alpha \mathrm{OHE} 1)$, and methoxy derivatives, represent the majority of either urinary or intratumor estrogens in human breast cancer patients, whereas they are relatively scarce the in urine or mammary glands of healthy women is strongly suggestive of the implication of these estrogen metabolites in the initiation and/or promotion steps of breast carcinogenesis. Furthermore, our data corroborate the results obtained by studies by Joachim Liehr, ${ }^{19}$ Ercole Cavalieri, ${ }^{20}$ and $\mathrm{H}$. Leon Bradlow ${ }^{21}$ revealing that $4 \mathrm{OHE} 2 \mathrm{and} / \mathrm{or} 16 \alpha \mathrm{OHE} 1$ are elevated in breast tumors as compared to normal mammary tissues, hence implying their role as mutagens. 
Overall, the data presented in this volume, including our own, combined with those accumulating in the literature, provide convincing evidence of the competence of estrogens as complete carcinogens (both initiators and promoters) in the human breast. Yet our recent studies on intratumor estrogens and the survival of breast cancer patients indicate that the potential role of individual estrogens in the onset and the clinical progression of breast cancer should be kept separate, because distinct estrogen derivatives may have diverse, if not opposite, roles in carcinogenesis and the progression of this malignancy. There is now substantial indication that estrogens can be implicated in the initiation, promotion, and progression of human breast cancer.

\section{ACKNOWLEDGMENTS}

This work was partly supported by the Italian Association for Cancer Research (AIRC) and the National Research Council (CNR).

\section{REFERENCES}

1. Castagnetta, L., G. Paparopoli, A. Traina, et al. 1977. Human breast cancer: studies on urinary excretion of endogenous oestrogens and its changes after hormone treatment. In Prevention and Detection of Cancer. Part I. H. Nieburgs, Ed. 1: 627636. Marcel Dekker. New York.

2. Castagnetta, L., G. D'Agostino, M. Lo Casto, et al. 1981. Breast cancer: a comparison of response to endocrine therapy and oestrogen excretion patterns including unusual metabolites. Br. J. Cancer 44: 670-674.

3. Castagnetta, L., G. D’Agostino, O.M. Granata, et al. 1983. Studies on estrogen status and estrogen hormone sensitivity in advanced endometrial cancer. In Steroids and Endometrial Cancer. M. Jasonni et al., Eds.: 233-236. Raven Press. New York.

4. Castagnetta, L.A., O.M. Granata, M. Lo Casto, et al. 1991. Simple approach to measure metabolic pathways of steroids in living cells. J. Chromatogr. 572: 25-39.

5. Castagnetta, L., O.M. Granata, L. Polito, et al. 1994. Different conversion metabolic rates of testosterone are associated to hormone-sensitive status and -response of human prostate cancer cells. J. Steroid Biochem. Mol. Biol. 49: 351-357.

6. Castagnetta, L.A., O.M. Granata, R. Farruggio, et al. 1995. Oxidative and reductive pathways of estrogens in hormone responsive and non-responsive human breast cancer cells in vitro. J. Steroid Biochem. Mol. Biol. 53: 367-374.

7. Castagnetta, L.A., A.M. Montesanti, O.M. Granata, et al. 1995. 17ß-Hydroxysteroid dehydrogenase activity in endometrial cancer cells: different metabolic pathways of estradiol in hormone-responsive and non-responsive intact cells. J. Steroid Biochem. Mol. Biol. 55: 573-579.

8. Castagnetta, L.A., O.M. Granata, A. Traina, et al. 2002. Tissue content of hydroxyestrogens in relation to survival of breast cancer patients. Clin. Cancer Res. 8: $3146-3155$.

9. Rogan, E.G., A.F. Badawi, P.D. Devanesan, et al. 2003. Relative imbalances in estrogen metabolism and conjugation in breast tissue of women with carcinoma: potential biomarkers of susceptibility to cancer. Carcinogenesis 24: 697-702.

10. Pasqualini, J.R., C. Gelly \& B.-L. Nguyen. 1990. Metabolism and biological response of estrogen sulfates in hormone-dependent and hormone-independent mammary cancer cell lines. Ann. N.Y. Acad. Sci. 595: 106-116.

11. Adams, J.B., R. VRahimis \& N. Phillips. 1992. Regulation of estrogen sulfotransferase by estrogen in MCF-7 human mammary cancer cells. Breast Cancer Res. Treat. 22: $157-161$. 
12. Castagnetta, L., O.M. Granata, M. Lo Casto, et al. 1986. Estrone conversion rates by human endometrial cancer cell lines. J. Steroid Biochem. 25: 803-809.

13. Miller, W.R. \& J.S. O’NEILl. 1987. Mammary steroidogenesis: therapeutic implications. Nuclear Med. Biol. 14: 369-376.

14. Adams, J.B., N.S. Phillips \& R. Hall. 1988. Metabolic fate of estradiol in human mammary cancer cells in culture: estrogen sulfate formation and cooperativity exhibited by estrogen sulfotransferase. Mol. Cell. Endocrinol. 58: 231-242.

15. Pasqualini, J., B. Schatz, C. Varin \& B. Nguyen. 1992. Recent data on estrogen sulfatases and sulfotransferases activities in human breast cancer. J. Steroid Biochem. Mol. Biol. 41: 323-329.

16. Castagnetta, L.A., O.M. Granata, F.P. Arcuri, et al. 1992. Gas chromatography/ mass spectrometry of catechol estrogens. Steroids 57: 437-443.

17. Castagnetta, L., S. D’Aquino, F. Labrie \& H. Bradlow, Eds. 1990. Steroid Formation, Degradation, and Action in Peripheral Tissues. Ann. NY Acad. Sci. 595.

18. LABRIE, F. 1991. Intracrinology. Mol. Cell. Endocrinol. 78: C113-C118.

19. LieHR, J. 2000. Is estradiol a genotoxic mutagenic carcinogen? Endocr. Rev. 21: 4054.

20. Cavalieri, E., D. Stack, P. Devanesan, et al. 1997. Molecular origin of cancer: catechol estrogen-3,4-quinones as endogenous tumor initiators. Proc. Natl. Acad. Sci. USA 94: 10937-10942.

21. Bradlow, H.L., R.J. Hershcopf, C.P. Martucci \& J. Fishman. 1985. Estradiol 16 $\alpha$ hydroxylation in the mouse correlates with mammary tumor incidence and presence of murine mammary tumor virus: a possible model for the hormonal etiology of breast cancer in humans. Proc. Natl. Acad. Sci. USA 82: 6295-6299. 